\title{
Orígenes del desarrollo comunal institucionalizado en Costa Rica
}

\author{
Miguel Ángel Mondol Velásquez \\ Consejo Institucional de Investigación, Vicerrectoría de Investigación, Universidad Estatal a Distancia, San José, Costa Rica; mmondol@uned.ac.cr
}

Recibido 18-I-2010 Corregido 19-II-2010 Aceptado 1-III-2010

\begin{abstract}
Origins of institutionalized community development in Costa Rica. Institutionalized community development in Costa Rica evolved within the "inward-oriented development model," driven by an "Epistemic Network" centered at the Economic Commission for Latin America. The model was designed to replace structures and non-rational social relations inherited from the "outward growth model" (agricultural exporter model). By the late 1970s, countries that implemented the model suffered a foreign debt that minimized the possibility of state intervention in economic growth. In Costa Rica, along with the fall of model, the institutionalized program declined, but during its first ten years, the program of community development through Development Associations had been efficient as a companion to modernization of agriculture and industry. Associations were widespread geographically, but concentrated in the border areas where the population was moving. They channeled state funding and local resources for road works and infrastructure that provided public services. With the crisis, the associations were criticized. The political leadership accused them of politicking; the social-democratic intellectuals of having raised no major national issues; and the academic left of having abandoned the effort of popular participation. But the associations had other goals, and they were successful in reaching them.
\end{abstract}

\section{KEY WORDS}

Community organizations, Cota Rica history, development, economic models.

\section{RESUMEN}

El desarrollo comunal institucionalizado surgió en Costa Rica articulado al "modelo de desarrollo hacia adentro", impulsado por una "Red Epistémica" centrada en la Comisión Económica para América Latina. El modelo pretendía sustituir estructuras y relaciones sociales no-racionales, heredadas del "modelo de crecimiento hacia fuera", o agroexportador. Hacia finales de la década de 1970 los países que implementaron el modelo sufrían un endeudamiento externo que minimizaba las posibilidades de intervención del Estado en las tareas del crecimiento económico. En Costa Rica, junto a la caída del modelo decayó el desarrollo comunal institucionalizado, pero durante sus primeros diez años, el programa de desarrollo de la comunidad, a través de las Asociaciones de Desarrollo había resultado muy eficiente como acompañante del proceso de modernización productiva en el agro y en la industria en el país. Las Asociaciones se habían extendido geográficamente, pero de manera especial hacia las mismas áreas fronterizas adonde se desplazaba la población. Con la crisis surgieron las críticas contra la Dirección Nacional de Desarrollo Comunal. La dirigencia política le acusaba de politiquería. La intelectualidad social-demócrata de no haber planteado los grandes temas nacionales. La izquierda académica de haber abandonado el esfuerzo por la participación popular. Pero la Dirección y sus Asociaciones surgieron dentro de otra agenda, para otros propósitos en cuyo cumplimiento fueron exitosos.

\section{PALABRAS CLAVE}

Organizaciones comunales, historia de Cota Rica, desarrollo, modelos económicos.
Costa Rica es un país centroamericano en el que han funcionado Asociaciones de Desarrollo Comunal articuladas con una institución nacional de origen estatal. En febrero del 2010 se celebraron 42 años de la constitución de la primera Asociación de Desarrollo en la bucólica comunidad de Santa Eulalia de Atenas. Hoy son más de tres mil, distribuidas a lo largo y ancho del país.
En promedio, en cada uno de los distritos administrativos debería haber unas ochenta de ellas.

Las Asociaciones nacieron con la Ley 3859 del 7 de abril de 1967 la cual constituyó a la Dirección Nacional de Desarrollo de la Comunidad (DINADECO) como institución responsable de la promoción, constitución, asesoría técnica y canalización de recursos hacia las Asociaciones 
de Desarrollo. En sus inicios DINADECO estuvo adscrita al Ministerio de Gobernación, Policía y Gracia.

\section{Antecedentes}

Una investigación previa, apoyada por la Vicerrectoría de Investigación de la UNED nos permitió (Mondol 2007):

- Establecer el contexto dentro del cual surgieron, hace 42 años, las Asociaciones de Desarrollo de la Comunidad.

- Describir el quehacer de DINADECO Y de las asociaciones en los primeros diez años de su existencia.

En términos muy breves, la investigación estableció aquel contexto de la siguiente manera:

El desarrollo comunal institucionalizado surge al interior de un proceso de acelerada transformación de las relaciones sociales. Por ejemplo, las Juntas Progresistas. Este proceso se había iniciado en la mitad del siglo pasado y concluyó, aproximadamente, en la década de los ochenta. El proceso, conocido como "desarrollo hacia adentro", implicó:

- Modernización del sector agropecuario.

- Industrialización por sustitución de importaciones.

- Diversificación del sector servicios.

- Crecimiento del aparato estatal creando condiciones para que se dieran los tres puntos anteriores.

No se crea que fue un proceso lento o superficial. Fue como se dijo rápido y profundo. Comparado con el conjunto de países latinoamericanos. Fue de los más acentuados (BID 1979).

De igual forma, fueron notorias y notables las consecuencias sociales de este proceso de desarrollo hacia adentro (Fig. 1):

- Concentración del ingreso en el mundo rural.

- Inmigración campesina hacia el Área Metropolitana de San José.

- Crecimiento de la pobreza.

- Emergencia de los primeros "grandes tugurios" en la capital, como resultado de un agudo déficit habitacional directamente relacionado con la magnitud de las corrientes inmigratorias.

Pero como se dijo, la primera investigación también describió lo que hicieron las Asociaciones y DINADECO en esos primeros años. Brevemente:

- Se constituyeron casi mil asociaciones sobre todo en la región periférica del país.

- Recibieron un fuerte apoyo financiero del Estado para la construcción de obras de vialidad (caminos, puentes)

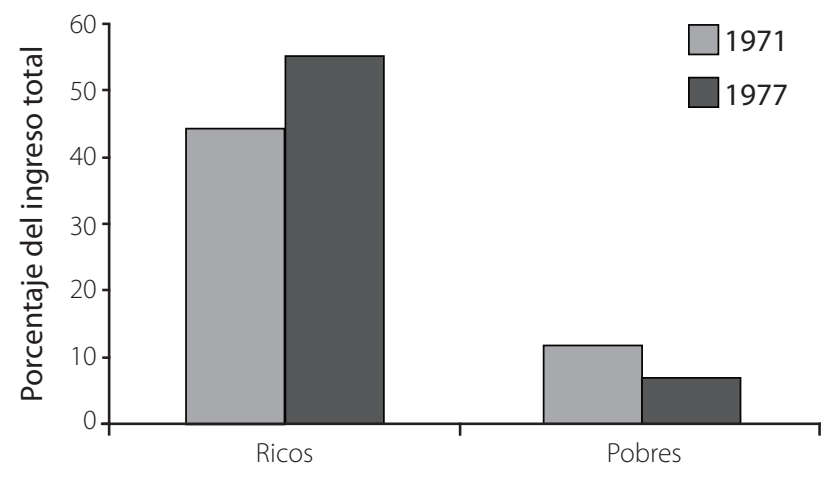

Nivel económico de las familias

FIG. 1. Distribución del ingreso en el mundo rural, 20\% de las familias más ricas y $30 \%$ de las familias más pobres (1971-1977)

y de infraestructura comunitaria (escuelas, centros de salud, salones comunales, plazas deportivas)

- Nació una dirigencia comunal muy relacionada con el Estado y que pronto ocupó puestos de elección popular en las municipalidades respectivas.

\section{Aspectos téorico-metodológicos}

Sin embargo, en el transcurso de esta primera investigación nos incomodaron las siguientes preguntas:

En un contexto de rápidas transformaciones económicas y sociales ¿para qué surgen estas Asociaciones de Desarrollo? ¿cuál fue su cuna conceptual? y, ¿porqué esa y no otra? Y en términos prácticos: ¿cuál es el sentido, el significado de su quehacer durante la primera década de su existencia?

Esas preguntas trataron de ser respondidas en esta segunda investigación también realizada gracias al apoyo de la Vicerectoría de Investigación de la UNED. Así es que el objetivo de la investigación se encaminó hacia la identificación de los factores explicativos que incidieron para el surgimiento del desarrollo comunal institucionalizado en Costa Rica.

Nuestro punto de partida fue el siguiente: En Costa Rica, el desarrollo comunal institucionalizado surge y funciona, por lo menos durante la década bajo estudio 1968-1978, articulado a un modelo de crecimiento que impulsó la Red Epistémica CEPAL en América Latina, después de la segunda post-guerra mundial.

La ley 3859 crea a DINADECO y a las Asociaciones en un acto simbiótico. En el mismo parto nacen la madre y las hijas. DINADECO supondría a las Asociaciones; éstas 
a DINADECO. Así es que, desde el inicio, convendría abordar el estudio de las Asociaciones como instancias de mediación entre el Estado y la sociedad civil. Veamos, de manera suscinta, algunos conceptos teóricos relacionados con esta mediación y en los cuales nos apoyamos durante el proceso investigativo.

- El Estado nace, en un momento histórico determinado, de las contradicciones de clase en la sociedad civil. Sin embargo, en la dinámica histórica de las sociedades, el estado "aparece" como teniendo existencia propia, independiente de la sociedad civil que le dio origen. El Estado es una apariencia que cobra existencia.

- Utilizando esta esquizofrénica imagen (Estado y sociedad civil como entes separados) el investigador costarricense, Sojo (2000) asume que:

"El Estado y la sociedad se relacionan en un ambiente de circularidad, en donde las actividades de uno influyen y determinan las actividades del otro, sin que exista una relación de primacía ni superposición. Desde el punto de vista de la sociedad, el elemento central de esta relación tiene que ver con el problema de la agregación de las demandas sociales; en otras palabras, la sociedad se relaciona con el Estado a través de demandas y lo fundamental para la construcción de esas demandas es la capacidad de la sociedad para agregarlas... Por su parte el Estado, el ámbito o término de la relación con la sociedad se da a través de políticas."

- Desde esa perspectiva, que no necesariamente compartimos pero que es metodológicamente útil para esta parte de la investigación, el desarrollo comunal institucionalizado podría ser visto como una política social que precisamente procura organizar las demandas de las comunidades costarricenses. En realidad la comunidad no es un todo homogéneo, en ella viven y se reproducen las contradicciones de clase que caracterizan a toda la sociedad.

- Recordemos que una política social frecuentemente se conceptualiza como la construcción racional de lo social dentro del Estado. El adverbio racional proviene Weber quien consideró al Estado moderno la forma legal-racional más adecuada para la administración de la sociedad capitalista.

"La cosa es muy distinta en el Estado racional, el único en el que puede prosperar el capitalismo moderno. Se funda en la burocracia profesional y en el derecho racional". (Weber 1944)

- Pero, ¿qué sucede cuando las relaciones entre el Estado y la sociedad civil se transforman en no-racionales? El investigador costarricense Roy Rivera, había puesto sobre el tapete este problema. En resumidas cuentas decía Rivera que, en Costa Rica, el régimen municipal había sido la forma privilegiada de relación entre el Estado liberal -el concepto es utilizado por él-y la sociedad civil local. Que esa forma había entrado en crisis con el Estado Social -también es de él este uso- .Es decir, la mediación entre Estado y sociedad civil se había transformado en no-racional. Por lo tanto, habría que suponer en adelante nuevas formas racionales de relación. Nos adelanta don Roy que una posibilidad era una política social que creara a las Asociaciones de Desarrollo de la Comunidad.

\begin{abstract}
"De esta forma, los espacios de actuación que anteriormente poseían (las municipalidades) se ven taponados, produciéndose una inmovilización de hecho del poder local municipal. Así lo nacionalestatal se va estructurando por encima de "lo local" y la relación entre la municipalidad y la comunidad se ve sustituida por una relación directa entre esta última y el Estado". (Rivera 1995)
\end{abstract}

Ahora bien, la formación y transformación de políticas sociales como las que crearon al desarrollo comunal institucionalizado ocurren, en América Latina, desde la "exterioridad" y desde "la interioridad", utilizamos interioridad y exterioridad como dos espacios dialécticamente interrelacionados. Es decir, desde la articulación entre agendas e intereses nacionales, con agendas e intereses internacionales (Lechner 1977). Este es un tema que ha desarrollado Juliana Martínez. En lo específico ella construyó dos conceptos que nos ayudaron durante la investigación:

- El concepto de "intereses nacionales"

"El principal foco de atención de esta aproximación al problema de la formación y la transformación de las políticas sociales es comprender cuáles son las configuraciones de poder e interés que han promovido la emergencia de ciertas políticas; cuál es la relación entre intereses particulares y acciones del Estado en tal o cual área de política social; y qué tipo de acción colectiva fue llevada a cabo para lograrlo. Estamos frente a grupos de presión, grupos de interés, coaliciones, vetos". (Martínez 2000)

- El concepto de agendas

“El énfasis está puesto en el conocimiento como variable independiente (y no solo resultante) de la formación y la transformación de las politicas sociales. Frecuentemente se vuelve la mirada al papel de actores generadores de agendas tales como organizaciones internacionales o grupos de técnicos y profesionales". (Martínez 2000) 
- Sabemos que las agendas generalmente se traducen en discursos. Pero no todos los discursos son discursos. Se producen y circulan por comunidades epistémicos situadas en una situación de poder/saber; saber/poder (en el sentido utilizado por Foucault). Así que otro de los conceptos metodológicos importantes fue el de discurso. Lo definimos como un conjunto coherente e interdependiente de nociones y conceptos relacionados con un saber especializado y enunciado y circulado desde una posición de poder por una red epistémica.

- Nos interesaron aquellos discursos que son producidos y circulados por redes epistémicas es decir por agentes institucionales quienes desde una posición de poder (saber/poder) producen, reproducen y circulan, durante un periodo estable un saber coherente y especializado que tiene raíces comunes.

- Finalmente algo sobre el procedimiento de la investigación: Siguiendo a Solano (2001) diremos que nuestra estrategia metodológica consistió en recorrer parcialmente la geografía de los discursos construido por redes epistémicas, posicionando la mirada en aquellos nodos conceptuales que se relacionan con una hipótesis general, previamente construida a la luz de enfoques teóricos aproximativos.

- Fue con estas pocas pero eficaces luces teórico-metodológicas, que en los meses de abril 2008 a marzo 2009 buscamos los factores explicativos para el surgimiento del desarrollo comunal institucionalizado en Costa Rica.

\section{Notas sobre un primer discurso acerca del desarrollo comunal institucionalizado}

Un primer discurso explicativo sobre el origen del desarrollo comunal institucionalizado fue elaborado y circulado hacia finales de la década de los setenta por una pequeña y difusa red epistémica de jóvenes académicos universitarios militantes en movimientos de la izquierda costarricense. Este discurso estuvo constituido por tres premisas. DINADECO y las Asociaciones surgen en el país, como:

- Tácticas de un partido político. El desarrollo comunal institucionalizado surge como una táctica del partido Liberación Nacional para apropiarse de los espacios locales de poder, particularmente allí donde tendía a poder escaños en la municipalidad.

- Táctica de un gobierno para destruir las Juntas Progresistas, valoradas en ese discurso como verdaderas formas de expresión popular.

- Parte de una estrategia imperialista para evitar la subversión armada como en otros países centroamericanos.
Esta red epistémica creó, entonces, un discurso simple, coherente y en esa medida propicio para su reproducción. Tanto que todavía hay académicos que siguen considerando esas premisas como verdades (Alvarenga 2005).

En la primera parte de la investigación demostramos la fragilidad de este discurso y en consecuencia, de esas tres premisas. No se trata aquí de reiterar argumentos. Baste decir que el esfuerzo estatal para constituir casi mil Asociaciones de Desarrollo en menos de una década y con una cobertura incluyente de la periferia geográfica nacional, ese esfuerzo -repetimos- es muy superior al necesario para lograr alguna o las tres premisas sobre las cuales descansa este discurso. Queremos decir, por ejemplo, que no hubiese necesitado el Estado costarricense hacer ese esfuerzo de crear DINADECO y casi un millar de Asociaciones para "destruir" a unas cuantas juntas progresistas, sobrevivientes urbanas de un gran movimiento del pasado, pero que ya para finales de la década de los sesenta empezaba a declinar. Una detallada argumentación contra estas premisas se dio en un texto anterior de Miguel Mondol (Mondol 2007).

\section{El discurso de la Red Epistémica CEPAL}

El discurso anterior no logró resolvernos las viejas preguntas que nos inquietaban. ¿Para qué surgen las Asociaciones? ¿cuál es el modelo conceptual que les da forma? ¿qué significado tiene su accionar durante la primera década de su existencia?

Hubo entonces que descubrir otro discurso que nos diera respuesta a estas preguntas. Provenía de lo que nosotros llamamos Red epistémica CEPAL.

\section{La red epistémica CEPAL}

No existe algo que se llame Red epistémica CEPAL. Es un constructo nuestro para designar al conjunto de actores institucionales e investigadores académicos que en la décadas de los cincuenta a setenta del siglo pasado formularon una interpretación diagnóstica y un discurso correlativo relacionados con el desarrollo y el subdesarrollo latinoamericano.

De manera más particular, Red epistémica CEPAL es un concepto ad hoc construído para referirnos a un conjunto de actores institucionales (AID, DESAL, CEPAL) y de académicos de universidades latinoamericanas (Germani, Véckemans, Medina Echavarría) interesados, por el tema del subdesarrollo latinoamericano durante las décadas de los sesenta y setenta. Por supuesto hubo otros actores individuales e institucionales, ocupados en el tema del subdesarrollo pero que no se vincularon específicamente al concepto de desarrollo de la comunidad. Su pensamiento 
no es estrictamente homogéneo. Por ejemplo, DESAL (1969) subrayaba la marginalidad como el problema central de los países latinoamericanos. La AID, la falta de actitudes modernas.

Este conjunto de actores institucionales y de académicos se expresaron, principal pero no exclusivamente, a través de la CEPAL (Comisión Económica para la América Latina), establecida en 1947 como agencia especializada de las Naciones Unidas y cuyos epónimos fueron Raúl Prebish, en lo económico y José Medina Echeverría en lo social.

Como sabemos, la CEPAL llegó a ser un centro de saber legítimo y especializado, en el sentido de que lo usa Foucault. Un saber especializado en el tema del desarrollo y el subdesarrollo latinoamericano y por lo tanto, capaz de producir un "discurso" sobre el tema del desarrollo y el subdesarrollo latinoamericanos desde una posición de poder/saber. Se emplea en la misma dirección que lo emplearon los estructuralistas franceses.. Así, este discurso circuló ampliamente y fue acogido por la mayoría de los países de la región. A ello contribuyó también la Organización de Naciones Unidas para la Ciencia y la Cultura (UNESCO 1962)

Mediante publicaciones conjuntas y una dinámica frecuente de reuniones y seminarios, estos actores lograron constituir una red epistémica dominante en el pensamiento social latinoamericano de la década de los cincuenta y sesenta. Para construir su discurso partían de una interpretación diagnóstica y de una agenda para implementar cambios en la región.

\section{Interpretación diagnóstica}

Detrás de la red subyace una interpretación diagnóstica de la realidad social latinoamericana.

a) En el tema económico, la red epistémica CEPAL, compartía la idea de uno de sus miembros, Raúl Prebish, de que el factor explicativo esencial del subdesarrollo latinoamericano era el deterioro creciente de los términos de intercambio, es decir, un balance negativo entre el índice de precios de lo que exportaban y de lo que importaban los países latinoamericanos hacia los países centrales o desarrollados. Esta idea tendía a radicalizarse cuando comprobaban que:

\footnotetext{
"Los cafés arábigos de América están siendo desplazados cada vez más por los cafés africanos de tipo robusto a medida que la bebida de preparación instantánea para la que se emplea un grano más barato, va cobrando mayor aceptación. El algodón mexicano tropieza con la competencia cada vez mayor de las fibras sintéticas. El caucho natural brasileño es ya cosa del pasado. El azúcar ya no constituye una base
}

sólida de ninguna economía de exportación". (UNESCO 1962)

b) Detrás y provocando tal deterioro en los términos de intercambio estaba una obsoleta estructura productiva agropecuaria, la cual en pleno siglo XX, presentaba características más de la hacienda colonial que de una empresa moderna. En relación con la industria - decía la red-seguía siendo mayormente artesanal. En consecuencia, decían ellos, la falta de racionalidad en esa estructura (en el sentido que Max Weber utilizaba este concepto) es lo que provoca el subdesarrollo latinoamericano.

c) Y en relación al Estado, argumentaban:

“En su aplicación a América Latina habría que tomar en cuenta ...la aptitud del Estado para superar la crisis social... por la falta de carácter representativo del proceso político y por otra, de la falta de responsabilidad en el proceso gubernativo" (UNESCO 1962)

d) En lo socialyen lopolítico-quees loque particularmente nos interesa-consideraba que aquí existía una:

- Dualidad estructural.

- Un marginamiento de la población más pobre, especialmente campesina.

- Sistemas institucionalizados de clientelismo y patronazgo propios de la hacienda colonial.

Estos factores serían ampliamente discutidos y criticados por la sociología crítica de los años setenta. Por ejemplo, contra el concepto de marginalidad que sustentaba esa Red, dos académicos, (Campanario \& Ritcher 1974) escribieron un excelente artículo que justamente denominaron "Superpoblación capitalista en América Latina: un intento de marginación del concepto de marginalidad. En la Red, sin embargo, siguieron diciendo que la marginación era un factor explicativo que impedía la transformación de las oligarquías latinoamericanas en élites modernas capaces y deseosas de impulsar los cambios en la estructura productiva. Más aún: la marginación política junto al viejo sistema del clientelismo reproducían una y otra vez en el poder a las desgastadas oligarquías latinoamericanas.

\section{Las agendas de la Red Epistémica CEPAL}

En lo que a la investigación interesa, convienen destacar dos agendas que manejó esta red durante las décadas 50 a 70 del siglo pasado. Una agenda relacionada con la implementación de un modelo de crecimiento o desarrollo en América Latina (modelo de crecimiento 
hacia adentro) y una agenda relacionada con el desarrollo comunal.

\section{Agenda para el modelo de desarrollo hacia adentro}

Habida cuenta de esta interpretación diagnóstica era necesario -según la red- implementar una agenda económica, que denominaron "modelo de crecimiento hacia adentro" que vendría a sustituir al "modelo de crecimiento hacia fuera", basado en la agro-exportación y que estuvo en vigencia en América Latina, según CEPAL desde la colonia hasta la segunda post-guerra mundial, cuando acusó graves síntomas de agotamiento. Proponía en su lugar CEPAL un modelo basado en la industrialización nacional por sustitución de importaciones, y en la modernización del aparato productivo agropecuario para la diversificación e incremento del valor agregado de las exportaciones. Esta propuesta económica era concordante con el pensamiento de los estructuralistas de CEPAL quienes veían en el deterioro creciente de los términos de intercambio una razón principal para el subdesarrollo de la región. Bien resumió así un académico costarricense la agenda de la red:

“El primer dogma es el del capital... el capital se convierte en la restricción predominante. El segundo dogma es el fundamentalismo industrial y agrícola, estrechamente relacionado con la inveterada idea de los economistas de asociar el desarrollo con industrialización. El tercer dogma lo constituye la sustitución de importaciones... Finalmente el cuarto y último dogma es el de la planificación... que abrirá las puertas al desarrollo". (Solano 2000)

En otras palabras: se pretendía una estricta racionalidad en el aparato productivo que coadyuvara a la disminución de los aspectos negativos en los términos de intercambio con el exterior.

“En el plano teórico ...el punto de partida de toda consideración se formula necesariamente en términos abstractos, como el de las relaciones entre la racionalidad económica y la racionalidad política; es decir, en qué forma y medida se exigen o repelen mutuamente sistemas económicos y sistemas políticos por virtud de la naturaleza y grado de racionalidad". (UNESCO 1962)

Así, en el fondo, las propuestas de la red epistémica CEPAL querían esto: impregnar de racionalidad a las relaciones sociales que prevalecían en la región latinoamericana. Y, para ellos, esta implementación de racionalidad debería llegar hasta el Estado.

Porque la pregunta práctica que se hacían era: quién acarrearía sobre sus hombros la ingente tarea de impregnar racionalidad al sistema económico, al sistema político y a la relación entre ambos... Allá, en los países centrales, en las europas, una burguesía industrial que le había arrebatado con las armas en la mano el poder a una decadente nobleza, bien.. Pero -argumentaban en la red- aquí no. Aquí en América Latina, la vieja oligarquía se negaba a convertirse en élite burguesa, moderna, progresista e industrializante. ¿Quién pues?

El Estado. Pero un estado caracterizado por una burocracia racional y moderna como en alguna ocasión lo había pretendido Max Weber. Quien por lo demás figuraba como el mentor más querido por los más destacados investigadores de la Red (UNESCO 1962).

\section{El discurso sobre desarrollo comunal en la Red Epistémica CEPAL}

Proponía entonces la Red la racionalización (modernización) de las relaciones sociales de producción, particularmente en el mundo rural de América Latina. Una racionalización que eliminara el cacicazgo y el paternalismo, herencias de la hacienda colonial. Una racionalidad que creara al "ciudadano del campo". Y al ciudadano de la ciudad, capaz de relacionarse con otros ciudadanos y con la institucionalidad del Estado, desplazando antiguas relaciones sociales patriarcales, de clientelismo, de patronazgo con las viejas oligarquías del continente.

Detrás y acompañando esa racionalidad subyacía un propósito de racionalidad política: destruyendo las bases de poder (la concentración latifundista del factor de producción Ilamado tierra) de las oligarquías latinoamericanas opuestas a la modernización del aparato productivo tanto agropecuario como industrial. Pero además, un propósito de racionalidad económica: hacer del campesino un "homus economicus", y por lo tanto coadyuvante en la urgente ampliación de la demanda interna para bienes que produciría la industrialización sustitutiva (lavadoras, televisores, etc).

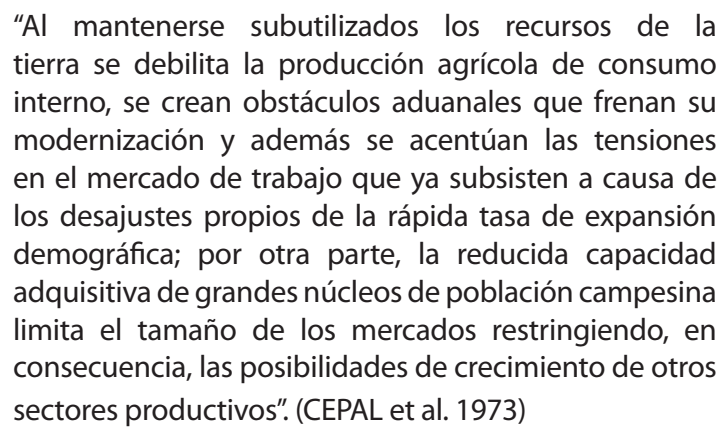

Es bajo tales consideraciones que surge la otra agenda de la red, la agenda sobre desarrollo de la comunidad, impulsada por la Red Epistémica CEPAL. Dentro de ella 
hay diferentes enfoques según el actor institucional del que se tratara. Esquemáticamente, el AID proponía un desarrollo comunal focalizado en lo local y en el cambio de actitudes en la población mediante el ejemplo de líderes comunales. DESAL enfatizaba la participación popular integrada dentro de la institucionalidad estatal; CEPAL veía el desarrollo comunal como una estrategia estatal relacionada con procesos de planificación económica y social, particularmente involucrados con la modernización de la producción agropecuaria y dentro de ésta, con la reforma agraria.

En síntesis, la Red consideraba que prevalecían en América Latina y particularmente en su mundo rural, formas no-racionales. En consecuencia proponía: a) racionalidad para el aparato productivo. b) racionalidad en las relaciones sociales que surgían del proceso productivo. c) racionalidad política y articulación racional entre lo político, lo social y lo económico. Desarrollo comunal sería una estrategia estatal coadyuvante para tales fines. Detrás de la propuesta de la Red, dándole respaldo teórico, se encontraba la concepción de Max Weber de un Estado moderno sustentado sobre una burocracia técnica, dueña de un saber especializado y capaz de ejercer el tipo de dominio que más conviene a la expansión capitalista: la dominación legal-racional.

\section{El desarrollo comunal institucionalizado en Costa Rica}

Con sus dos agendas bajo el brazo, (la del crecimiento hacia adentro y del desarrollo comunal institucionalizado) los expertos de la CEPAL se lanzaron por los caminos de América Latina para convencer a moros y cristianos (es decir a gobiernos de toda índole) sobre las bondades que sobrevendrían si practicaban dichas agendas.

Así, en Costa Rica y en Centroamérica, durante las décadas de los sesentas y setentas, hubo una participación muy activa de expertos de la Red, y en particular de CEPAL, para implementar el modelo de crecimiento hacia adentro: modernización del aparato agropecuario; industrialización sustitutiva y creación de una herramienta vital para los dos propósitos anteriores: El Mercado Común Centroamericano.

Articulado con este proceso de implementación del modelo de crecimiento hacia adentro los expertos de la Red Epistémico proponían la fórmula, la agenda, del desarrollo comunal institucionalizado. Hubo una participación directa y eficiente de la Red para la puesta en marcha de programas nacionales de desarrollo comunal institucionalizado en varios países de la Región. (Guatemala 1964; Chile 1964; Costa Rica, 1967; El Salvador, 1968; Panamá 1969 No se trató solo de un acompañamiento teórico o conceptual. Fue una presencia activa, a través de expertos, quienes trabajando con profesionales del aparato público construyeron dichos programas.

En el caso de Costa Rica, ocurrió desde 1958 cuando expertos de la Red epistémica CEPAL, iniciaron el diseño de lo que sería en desarrollo comunal institucionalizado en Costa Rica.

De las consideraciones precedentes surgió el punto de partida de lo que habíamos establecido al principio de estas notas: En Costa Rica, el desarrollo comunal institucionalizado surge y funciona (por lo menos durante la década bajo estudio 1968-1978) articulado a un modelo de crecimiento que impulsó la Red Epistémica CEPAL en América Latina, después de la segunda postguerra mundial. Y las características de ese modelo de desarrollo comunal institucionalizado se fundamentan en la búsqueda de una racionalidad en las relaciones entre el Estado y la sociedad civil local. Una racionalidad provocada desde el Estado.

\section{El "interés nacional"}

Surge aquí otra de esas preguntas inquietantes: $\mathrm{Si}$ esa Red, promovió su agenda sobre el desarrollo comunal, simultáneamente en otros países, ¿porqué aquí, en Costa Rica, la concreción de esa agenda adquirió características tan particulares, muy disímiles a las que adquirió, para citar dos ejemplos, en Guatemala y en El Salvador? .

Viene entonces en nuestra ayuda Juliana Martínez quien nos vuelve a recordar que, junto a las agendas internacionales están lo que ella denominó "intereses nacionales", es decir "las configuraciones de poder e interés que han promovido la emergencia de ciertas políticas" (Martínez 2000)

En lo que atañe al desarrollo comunal institucionalizado costarricense ese "interés nacional" fue reconocido, elaborado y circulado en un discurso de otra red epistémica que en la década de los sesenta y setenta del siglo pasado presentaba-cuando menos- las siguientes características: Un conjunto de actores nacionales, jóvenes, egresados de las primeras promociones de las carreras de ciencias sociales en la Universidad de Costa Rica (trabajo social, economía, sociología) institucionalizados en la burocracia pública, particularmente en las áreas de educación y salud. Conocían -por su trabajo- muy bien el país y tenían una visión clara de lo que para ellos debería ser el desarrollo nacional. En su mayoría eran jóvenes socialdemócratas confesos y convencidos de la "utopía tecnológica" promovida por Figueres y del "Estado Empresario" de Daniel Oduber. Hubo, en su momento, algunos y algunas ubicadas un poco más hacia la izquierda, saboreando la 
idea un "socialismo con sello a la tica") Les aglutinaba su quehacer laboral, pero también seminarios, reuniones y publicaciones conjuntas del Centro de Estudios Democráticos para América Latina (CEDAL) ubicado en las montañas heredianas y en donde vivía otro mentor del grupo y quien sería Presidente de la República pocos años después: Luis Alberto Monge Álvarez.

Este grupo de "interés nacional" elaboró y circuló un discurso que partía de tres premisas:

a) Había -según ellos- una nueva Costa Rica en la cual se podía constatar un extraordinario crecimiento del sector agropecuario, industrial y de la actividad Estatal.

Los siguientes datos dicen acerca de la forma cómo se implementó el modelo de crecimiento hacia adentro, en Costa Rica, durante el periodo que nos ocupa en esta investigación. Para nosotros esta información es relevante pues, repetimos, es el marco dentro del cual surge la propuesta del desarrollo comunal en el país. Los datos parecían confirmar esta presunción.

Si para 1950 menos del $20 \%$ del territorio nacional estaba dedicado a la producción agropecuaria, entre 1950 y 1976 casi se duplicó el número de hectáreas dedicadas a la agricultura y se triplicó el número de hectáreas dedicadas a la ganadería. Además, en el agro, se puso en marcha "la utopía tecnológica de Figueres": El capital instalado por persona ocupada en el sector agropecuario creció un 60,5\% sólo entre 1963 y 1973 (Reuben 1982)

Debido principalmente a esa tecnificación del campo, la tasa de crecimiento anual en el valor agregado del sector agropecuario, durante el periodo 66-70 fue tres veces superior a la observada para el conjunto de países latinoamericanos (Reuben 1982).

En relación con la industria -la otra vertiente del modelo de crecimiento hacia adentro- sabemos que entre 1964 y 1975 la productividad media en todas las ramas industriales creció a una tasa geométrica anual del 7,21\%. Esa tasa fue mayor en las industrias no-tradicionales (Reuben 1982).

Durante el largo periodo 1961-1977, la tasa de crecimiento anual en el valor agregado del sector manufacturero fue, en Costa Rica, siempre superior a la observada en América Latina (Reuben 1982).

El Estado fue impulsor directo de todo este proceso de crecimiento industrial y agropecuario. Según datos aportados por Vega Carballo, entre 1950 y 1970, "el sector público continuó teniendo una expansión sin precedentes que no pudo ser superada por el sector privado...En 1950 el gasto público ascendía a un 33\% del PIB; en 1960 la cifra del 55,6\% y en 1970 la cifra alcanzó a 67,4\%" (Vega s.f.) b) Surgiría una fracción industrial que algunos académicos la describían como clase social:

"La principal consecuencia sociopolítica de la consolidación de la base industrial que se vive en nuestro país en el periodo de posguerra, es la configuración de un grupo claramente industrializante. Contrario a lo que se ha dicho, no se trata de simples empresarios aislados, incapaces de conformar una ideología y un proyecto propios, sino que se trata efectivamente de una burguesía industrial, que si bien se muestra débil y timorata en comparación a su homónima de los países desarrollados, muestra también claramente el deseo de imponer su proyecto de desarrollo nacional". (Herrero \& Garnier 1981)

\section{c) El cambio del modelo de Estado}

Tal fue el crecimiento del aparato estatal y su rol en la actividad económica y social del país (Fig. 2), que para finales de la década de los setenta, algunos académicos costarricenses pensaban que estas cifras revelaban nuevos rasgos en el Estado costarricense los cuales, eventualmente, le llegarían a diferenciar del otrora Estado liberal, esta idea ha sido bastante discutida y criticada por excelentes académicos como Manuel Solís, cuya referencia está en la bibliografía de este artículo Estos académicos sugerían que podríamos estar caminando hacia un "capitalismo de estado" o, como le denominaban otros, "Capitalismo de Estado-benefactor" (Herrero \& Garnier 1981).

\footnotetext{
"Se puede hablar a partir de este periodo (1970 en adelante) de un estado empresario por la importancia económica y política que cobraron las empresas productivas creadas por el Estado (CODESA). Se destaca también en este momento una tendencia a la centralización de tareas en el poder ejecutivo, en contraposición con la descentralización que marcó el periodo anterior...Además el Estado de este periodo amplía su presencia en el ámbito de la reproducción de la mano de obra" (Vega 1983).
}

d) En síntesis, este era el clima económico y sociopolítico prevaleciente en la década de los setenta, tan nítido, tan vívido que como hemos visto, el eco de ese clima llega hasta a los académicos universitarios de la década de los ochenta: Reinaba una visión nacionalista, optimista. Se decía que una nueva burguesía industrial, de consuno con nuevos sectores agrarios, pondrían en marcha a través de (y con) un fortalecido Estado nacional un proyecto de desarrollo nacional basado en la industrialización sustitutiva y en la modernización del aparato productivo agropecuario. Hasta la segunda mitad de la década de los setentas el proyecto desarrollista parecía que lograba lo que se había 
propuesto. Y es, repitámoslo una vez más, en ese marco y con ese clima optimista, cuando se generó el modelo del desarrollo comunal costarricense.

En absoluta conformidad con estas pautas optimistas y de transformación nacional correspondería al desarrollo comunal crear condiciones para la participación organizada e integrada en el Estado. Esta participación sería instrumental porque coadyuvaría a la puesta en marcha de las necesarias reformas que requería el mundo rural. Reformas que ya estaban siendo puestas en movimiento por una floreciente nueva clase social-agraria e industrial- la cual actuando junto a la tecnocracia de un Estado moderno y racional -a cuya cabeza estaría una Oficina de Planificación Nacional-prometía sacarnos del subdesarrollo.

\section{La construcción de un modelo de desarrollo comunal en Costa Rica}

Entonces, producto del encuentro entre la agendas de la Red Epistémica CEPAL junto con la agenda de interés nacional de una red interna, surge el modelo conceptual inicial del desarrollo comunitario institucionalizado en Costa Rica. Veamos ahora, en ese modelo, sus rasgos conceptuales y los resultados concretos de su aplicación en nuestro país.

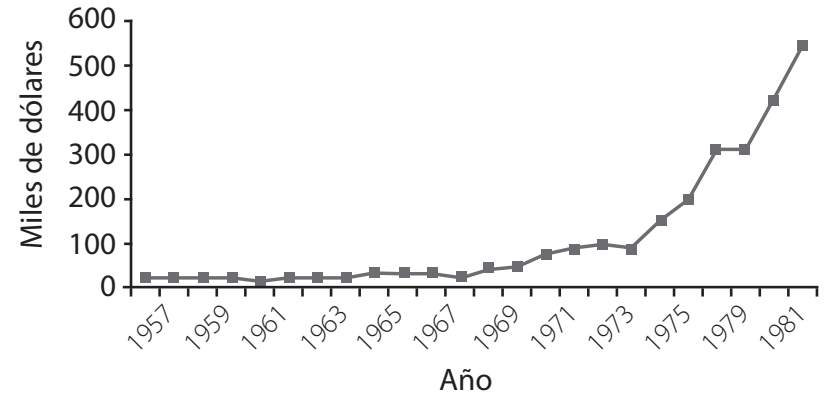

FIG. 2. Inversión pública total en Costa Rica, entre 1956 y 1982 (en dólares al tipo de cambio vigente en cada año).

\section{Aspectos conceptuales}

Igual que el modelo de crecimiento, el Programa para el desarrollo de la comunidad se inició mediante la participación activa de la CEPAL. Fue a esta Agencia de Naciones Unidas adonde recurrió la administración Echandi Jiménez, en su primer año de gobierno (1958) para analizar las posibilidades de implementar un Programa Nacional de Desarrollo de la Comunidad en el país.
Al año siguiente, 1959, la CEPAL conjuntó a un Grupo Nacional de Trabajo sobre Desarrollo de la Comunidad el cual funcionará como una "Red epistémica Nacional sobre Desarrollo Comunal" e hasta la mitad de la década de los setenta. Como se analizó en la primera parte de la investigación, fue una red conformada por profesionales jóvenes, social-demócratas en su mayoría, que venían trabajando en programas sectoriales de desarrollo comunal en salud y educación, principalmente (Mondol 2007)

Posteriormente, en 1965, la Agencia Internacional para el Desarrollo (AID), interviene en la red epistémica cuando ésta actúa -a través de la recién constituida Oficina para el Desarrollo de las Comunidades Rurales- en apoyo al Plan de Desarrollo Regional de la Península de Nicoya, cuyo principal dirigente fue el Lic. Armando Araúz, miembro desde el inicio de la red epistémico nacional y luego -1966- diputado proponente del proyecto de Ley que crearía a DINADECO. En 1970 la AID volverá a intervenir mediante la inclusión de DINADECO en el Primer Plan Agropecuario, financiado con fondos de esa agencia norteamericana. Parte de esos recursos se utilizaron para la asistencia técnica que brindó AITEC (Acción Internacional Técnica) a DINADECO durante el cuatrienio 70-73. Un fin primordial de la asistencia fue diseñar un sistema operacional para el trabajo sistemático, racional, de DINADECO con las Asociaciones (González 1977)

En 1966 y 1967 la participación de DESAL (Desarrollo Social para América Latina) fue importante para incluir en el proyecto de ley aspectos relacionados con la participación ciudadana en las Asociaciones de Desarrollo.

Como puede apreciarse, todos los actores institucionales de lo que aquí hemos venido llamando Red Epistémica CEPAL, (AID, DESAL, CEPAL) estuvieron muy presentes en la formulación del Programa de Desarrollo Comunal costarricense.

No existe, sin embargo, un sólo documento que manifieste lo que la red epistémica CEPAL quería en la formulación de ese Programa de Desarrollo Comunal. Existen pequeños documentos dispersos. Tampoco, por supuesto, referencias teóricas. Hay que inferirlas y ponerlas en relación con las premisas del modelo de crecimiento hacia adentro impulsado por esa misma red. Lo haremos a partir, exclusivamente, del documento más oficial del desarrollo comunal en Costa Rica: su ley constitutiva del 7 de abril de 1967.

\section{Desarrollo comunal: una estrategia}

En primer lugar estimamos que la Red Epistémica CEPAL, en asocio con esta red epistémica nacional, pensó al Programa de Desarrollo Comunal como una estrategia para el logro del modelo de crecimiento hacia adentro. 
Ello se desprende del primer artículo de la Ley constitutiva de DINADECO y las Asociaciones:

\begin{abstract}
"Artículo primero: Créase la Dirección Nacional de Desarrollo de la Comunidad con carácter de órgano del Poder Ejecutivo adscrito al Ministerio de Gobernación y Policía, como un instrumento básico de organización de las comunidades del país, para lograr su participación activa y consciente en la realización del Plan Nacional de Desarrollo Económico y Social" . (DINADECO 1974)
\end{abstract}

Con respecto a los contenidos de la estrategia, una posible definición conceptual del desarrollo comunal institucionalizado, para ese periodo y en Costa Rica, podría ser la siguiente:

Construcción en el Estado de una nueva relación racional/legal de carácter privilegiado entre el Estado y la sociedad civil local, relación fundamentada en el concepto de participación ciudadana para la consecución de objetivos planificados desde el estado y legitimados desde la sociedad civil local.

Veamos, en detalle, los conceptos esenciales de este enunciado:

\section{Construcción en el Estado}

Pese a las pretensiones de autonomía de las Asociaciones de Desarrollo de la Comunidad -autonomía que coyunturalmente fue apoyada por el mismo DINADECO, como en el periodo 70-73, en la administración institucional de José Luis González Ramos- legalmente la promoción, constitución, asesoría técnica, financiamiento, y disolución de las Asociaciones ocurre, en última instancia, a juicio discrecional de DINADECO. (artículo 2, inciso d, $\mathrm{g}$, i, k. Artículos 14, 15, 19, 25, 26 y 35 de la Ley).

\section{Nueva relación}

El estado costarricense a lo largo de su historia a construido múltiples mediaciones con la sociedad civil local: Juntas de Educación, Comités de Salud, Comités de caminos vecinales, entre otros. La Asociación de Desarrollo es una nueva forma de mediación, que no sustituye a las anteriores pero las supera en términos de mayor apoyo legal, financiero y técnico desde el Estado.

\section{Relación entre el Estado y la sociedad civil local}

DINADECO es una burocracia con un saber especializado (desarrollo comunal) que representa al Estado en la relación Estado-sociedad civil local. Esta relación -desde el punto de vista del Estado- es planificada en Planes Quinquenales de Desarrollo de la Comunidad. El Primer Plan Nacional de Desarrollo de la Comunidad correspondió al periodo 70-74 (Administración Figueres Ferrer). Este Plan se articuló -por lo menos formalmente- al Primer Plan Nacional Agropecuario (Tercero \& Salas 1983). El Segundo Plan Nacional de Desarrollo de la Comunidad se elaboró para el periodo 75-78 (Administración Oduber Quirós) y se relacionó con el Programa Nacional de Asignaciones Familiares.

Tómese nota, en relación con la idea de racionalidad en el Estado impulsada por la Red epistémico CEPAL, que DINADECO es una de las primeras instituciones sociales nacionales que planificó, a mediano plazo, su quehacer institucional. Ejemplo de ello son sus dos planes quinquenales que citamos en el párrafo anterior.

No se dice explícitamente, pero en esa estrategia, la Asociación de Desarrollo de la Comunidad supone a la sociedad civil local, es decir a la comunidad. Asociación y comunidad, en términos de DINADECO, resultaron sinónimos, porque se suponía que la Asociación representaba los intereses de toda la comunidad. En setiembre de 1972, DINADECO contrató a un consultor internacional, Benjamín Maluenda para institucionalizar la representatividad de la Asociación a nivel local. Cfr. Maluenda, Benjamín. "Hacia la coordinación nacional de politicas y relaciones operativas entre DINADECO y el Instituto de Fomento y Asesoría Municipal (IFAM) en José Luis González Ramos, 1977, página 189. Como veremos en otra ocasión, la identificación artificiosa que hizo DINADECO entre comunidad=asociación explica las dificultades de las Asociaciones para convocar a toda la comunidad en sus quehaceres. En este mismo sentido, el artículo 30 de la Ley sólo permitía la constitución de una Asociación de Desarrollo Integral por comunidad.

\section{Racional y legal}

En sus mediaciones anteriores, la relación del Estado con la sociedad civil local, era coyuntural, asistemática y personal entre el funcionario y la organización comunal. (por ejemplo, entre el funcionario municipal con una Junta de Educación). Ahora las relaciones serían legales porque hay un conjunto de normas específicas contenidas en la Ley 3859, pero sobre todo en su reglamento que regulan esta relación. Pero además, durante los primeros años de funcionamiento (periodo 70-74) DINADECO, con asistencia técnica, produjo un Manual de Promotores y un Sistema Operacional, basado en la administración por objetivos, con la intención de sistematizar, optimizar y homogenizar las relaciones entre sus funcionarios y las comunidades. Según González (1977), se enfrentó el problema de dotar al trabajador de campo de un marco metodológico de referencia de un sistema de trabajo que ordenara y focalizara su acción, y de un mecanismo para 
seleccionar las áreas geográficas y funcionales de trabajo de acuerdo con criterios claros y generales

\section{De carácter privilegiado}

El Estado otorga, de manera permanente, con recursos financieros a las Asociaciones de Desarrollo de la Comunidad. La ley 4860 de noviembre de 1971, reformó el artículo 19 de la Ley 3859, para que se leyera así:

“El estado incluirá en el Presupuesto Nacional una partida equivalente al dos por ciento del estimado del impuesto sobre la renta de ese periodo, el cual se girará al Consejo Nacional de Desarrollo de la Comunidad para las asociaciones de desarrollo de la comunidad debidamente constituidas y legalizadas. El Consejo Nacional de Desarrollo de la Comunidad depositará esos fondos en el Banco Popular y de Desarrollo Comunal para girar exclusivamente a las Asociaciones de desarrollo de la comunidad, y a la vez, crear un fondo de garantía e incentivos para financiar o facilitar el financiamiento de proyectos que le presenten las mismas asociaciones de acuerdo con la respectiva reglamentación". Ley 3859, artículo 19 reformado por la ley 4890 del 16 de noviembre de 1971

Desde esta perspectiva, las Asociaciones también podrían ser analizadas como el último eslabón de una cadena institucional del Estado.

Otros artículos de la Ley amplían las posibilidades de recursos estatales para las Asociaciones. (Artículos 19, 20 y 36 de la Ley).

\section{Participación ciudadana}

La Asociación fue considerada un mecanismo para estimular la participación de los ciudadanos (artículo 3, inciso b y artículo 16 de la ley) (Palma 1981)

Es una participación que puede estructurarse verticalmente (Artículo 41 de la Ley 3859).

Pero es una participación integrada al Estado. El inciso f) del artículo 3 de la ley, establece como objetivo de DINADECO, "planear y promover la participación activa y organizada de las poblaciones en los programas nacionales, regionales o locales de desarrollo económico y social"

\section{Objetivos planificados}

La Asociación de Desarrollo de la Comunidad es una organización que convoca a la participación ciudadana para fines previamente planificados, ya sean fines estatales o fines locales. En el primer caso, ya se dijo que el artículo 1 de la Ley 3859 , creaba a DINADECO,

“...como un instrumento básico de organización de las comunidades del país, para lograr su participación activa y consciente en la realización de los objetivos del Plan Nacional de Desarrollo Económico y Social" (artículo primero, ley 3859). En el mismo sentido se orienta el inciso f) del artículo 3: "planear y promover la participación activa y organizada de las poblaciones en los programas nacionales, regionales y locales de desarrollo económico y social"

En el segundo caso, es decir en relación con fines u objetivos locales, el artículo 32 de la Ley, establecía que "las asociaciones de desarrollo tienen la obligación de formular anualmente un programa de actividades y someterlo a conocimiento de la Dirección Nacional de Desarrollo de la Comunidad".

José Luis Vega Carballo, un académico cercano a lo que aquí hemos denominado Red Epistémica Nacional y quizá quien mejor se ha detenido a analizar el tema del desarrollo comunal en Costa Rica describía hace unos veinte años , conceptualmente, lo que él llama la primera etapa del desarrollo comunal. Esta descripción concuerda a grandes rasgos con lo que apuntamos en este capítulo:

"...la estrategia implicaba desde un principio, la intervención estatal en el orden comunal con el afán de aportar los recursos de éste al esfuerzo global por la modernización impulsada "desde arriba" ...El objetivo no era reforzar la tradición y el conservatismo de la vida local...sino romper su aislamiento y sujetarla al carruaje de los nuevos tiempos, lo cual por otra parte redundaría en la posibilidad de contar "hacia abajo" con buena cadena de transmisión de los impulsos desarrollistas sin caer por la vía centralizada en una imposición completa y alienante como la que afectaba al régimen municipal. Se trataba de un movimiento de doble vía pero se dejaba muy claro en el contexto y la letra de la ley, que los más fuertes estímulos del proceso renovador vendrían del "centro" hacia "la periferia", del pujante Estado y sus instituciones hacia las débiles localidades del interior del territorio..." (Vega 1987)

\section{RESULTADOS}

Durante este primer decenio de funcionamiento, ¿cómo se concretizó ese modelo conceptual?, en otras palabras: ¿qué hicieron las Asociaciones? Y, eso que hicieron, fue funcional o no para la agenda sustantiva que proponía la Red Epistémico CEPAL, es decir, el modelo de desarrollo hacia adentro?

\section{En relación con DINADECO}

Digamos, primero que entre 1968 y 1978 el ente burocrático especializado, DINADECO, creció de una manera 
extraordinaria: mayor presupuesto, sistematización de técnicas para atender a las Asociaciones, regionalización de las oficinas de DINADECO, etc.

A partir de 1970 DINADECO había incrementado sus ingresos. Sustancialmente durante el periodo 1970-1974. (Administración Figueres Ferrer) Vertiginosamente, durante el periodo 1975-1978 (Administración Oduber Quirós).

El primer incremento de ingresos fue producto de su inclusión en el Primer Plan Nacional de Desarrollo Agropecuario, financiado mediante crédito público con la Agencia Internacional para el Desarrollo (AID).

El segundo incremento -sustancial- fue por su inclusión en el Programa Nacional de Desarrollo Social y Asignaciones Familiares.

Para 1978, DINADECO había constituido por lo menos una Asociación en el $90 \%$ de los distritos administrativos en que subdividía el país.

Durante la primera fase del periodo bajo estudio (19701973) DINADECO invirtió a través de sus Departamento de Promoción y Adiestramiento grandes recursos para "concientizar" concepto que utilizamos aquí con el significado que tenía en el discurso revolucionario de los años setentas al dirigente comunal sobre los problemas no sólo de su comunidad sino del país. Estos esfuerzos no alcanzaron para que el dirigente comunal se transformara en "agente de cambio social" tal como lo quería la institución en aquellos años.

\section{En relación con las Asociaciones}

Desde el punto de vista de las Asociaciones de Desarrollo Comunal, entre 1968 y 1978, el desarrollo comunal institucionalizado se puede caracterizar de la siguiente manera de acuerdo con los datos empíricos que surgieron de la investigación. Las Asociaciones de Desarrollo de la Comunidad:

Se extendieron por todo el país. Prácticamente no hubo distrito administrativo sin por lo menos una Asociación.

Se ubicaron -sobre todo- en las áreas geográficas más periféricas, más distantes del centro del país, esto es, precisamente aquellas áreas que estaban experimentando fuertes movimientos de población producto de la expansión agropecuaria y la modernización productiva (Fig. 3). En el sector agropecuario buena parte de los cambios demográficos se relacionaron con la perviviencia de la estructura productiva latifundio capitalizado/minifundio. En el sector industrial con la concentración productiva en el Area Metropolitana de San José y cantones aledaños.

Fueron un instrumento válido, para trasladar recursos hacia las comunidades más periféricas. En este sentido, las ADC superaron la relación Estado-Municipio que había prevalecido durante lo que algunos llaman la época del Estado Liberal costarricense y que ya resultaba "disfuncional" para los requerimientos expansivos -sobre todo agropecuarios- del modelo de crecimiento hacia adentro.

Las ADC tenían fuentes de financiamiento externas: $2 \%$ (luego 1,25\%) del impuesto sobre la renta, ayudas en materiales del Ministerio de Obras Públicas y Transportes, crédito subsidiado con el Banco Popular, y por supuesto recursos generados internamente. Es muy posible que todos estos recursos se canalizaran hacia los mismos fines que los originados en partidas específicas, estas concentraron sus esfuerzos en (Cuadro 1):

- Obras de infraestructura, equipamiento y mejoramiento comunal (salones comunales, escuelas, centros de salud, plazas de deporte, urbanismo).

- Obras de vialidad (caminos, puentes, etc.).

- Obras para la dotación de agua y electricidad a las comunidades.

Gran parte de estas obras se ejecutaron en las áreas periféricas del país, y particularmente, en aquellas donde había una expansión agropecuaria modernizante según la Red epistémica (EPAL) o una expansión industrial capitalista dependiente.

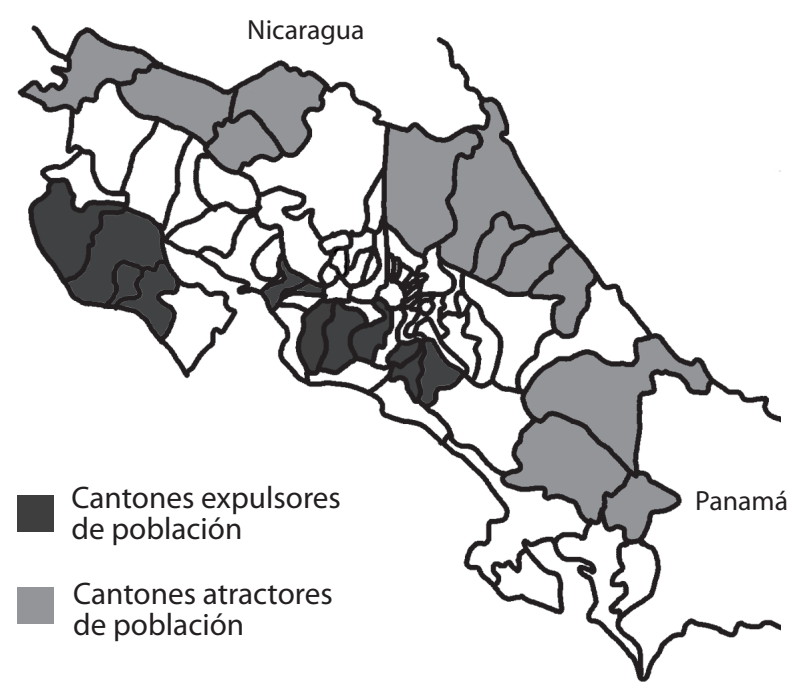

FIG. 3. Concentración (según densidad organizacional) de las Asociaciones de Desarrollo de la Comunidad de Costa Rica entre 1968 y 1978. 


\section{En conclusión}

Una función principal del desarrollo comunal institucionalizado fue acompañar los intensos procesos de expansión agropecuaria e industrial que experimentó el país en la década de los setentas.

Adicionalmente habría que reconocer también que las Asociaciones fueron un instrumento idóneo para reproducir la fuerza de trabajo agropecuaria e industrial en niveles más altos, mejores, que cómo se reprodujo en otros países latinoamericanos, $Y$ también para reproducir la ideología dominante, como lo han señalado Camacho (1979) y Palma (1981) aquí, la población que se movilizó hacia las nuevas zonas de colonización agropecuaria pudo contar $-y$ desarrollo comunal fue un instrumento eficaz para ello- con servicios básicos que de alguna forma reproducían aquellos que tenían en sus comunidades de origen: agua, electricidad, vivienda, escuela y salud. Y esto es válido también para los nacientes barrios situados alrededor de la zona industrial urbana.

\section{CUADRO 1}

Distribución (según fines) de las partidas específicas giradas a las Asociaciones por el Estado en el período 1970-1978

\begin{tabular}{lcc}
\hline \multicolumn{1}{c}{ Tipo de obra } & Región Central & Región Periférica \\
\hline Vialidad & 15,8 & 33,2 \\
Infraestructura & 43,5 & 35,8 \\
Varias & 21,7 & 11,5 \\
Subtotal & 81,1 & 80,5 \\
Otras & 18,9 & 19,5 \\
Total & 100,0 & 100,0
\end{tabular}

\section{El agotamiento del modelo: 1978}

Generalmente se señala a 1978, como el año en que inició el declive de DINADECO y las Asociaciones de Desarrollo Comunal. En ese año, la institución pierde su sitio de privilegio en el Ministerio de Gobernación y pasa a ser dependencia de un frágil y recién nacido Ministerio de Cultura. La dinámica de constitución de Asociaciones se pierde. Disminuyen los recursos hacia las Asociaciones, y no se volverá a hablar -hasta una década después- de mecanismos para estructurar verticalmente -en federaciones y confederaciones- a las Asociaciones de Desarrollo.
Con el agotamiento surgieron las críticas. En la celebración del décimo aniversario de la institución amigos y enemigos se conjuntaron para señalar más sombras que luces en ese pasado decenio.

Para la dirigencia política que apoyó y dirigió inicialmente a la institución, y la cual era adversa a la de Liberación Nacional,

"Los políticos desgraciadamente cambiaron la filosofía del desarrollo comunal la cual era, originalmente "hacer chocolate sin cacao". Es decir, que los pueblos resolvieran sus problemas mediante esfuerzo propio. Pero los políticos comenzaron en el propio gobierno de Trejos a cambiar las cosas; es decir, en vez de dejar que la gente hiciera un esfuerzo propio les llevaron partidas específicas y el dos por ciento sobre el impuesto de la renta... Entonces las Asociaciones se han dedicado a pedir y pedir, nada más". (Quesada 1977)

Para los profesionales socialdemócratas, responsables del inicio de la institución:

"Queremos hacer hincapié que aunque las comunidades mismas muchas veces no están concientizadas de sus necesidades, si la institución promotora del desarrollo comunal busca convertirse en una agencia de movilización social que no se contente con meros cambios cosméticos en las comunidades, tendrá que apoyar proyectos que tiendan a modificar las estructuras socio-económicas existentes para que alteren la situación de dependencia." (González 1977)

Y para la intelectualidad crítica:

"Lo anterior es posible gracias al escaso desarrollo político organizativo que muestran la mayoría de las Asociaciones de desarrollo comunal en el país...Esto queda evidenciado en la forma en que los dirigentes asumen el trabajo comunal. Una evaluación hecha por la misma DINADECO, da cuenta que el $86,4 \%$ de los participantes en Asociaciones no discuten, analizan ni deciden sino que se integran en tareas que ya han sido decididas en su nombre". (Aguilar 1983)

Sin embargo, habría que relativizar estas críticas. Son críticas válidas. Pero que hoy, a la luz de los resultados de esta investigación habría que replantearlas. Porque parecen referirse a un DINADECO y a unas Asociaciones que objetivamente pretendían otra cosa. Bien lo expresa un académico:

"Según muchos, el movimiento de desarrollo comunal y su principal institución rectora ha llegado a ser por medio de estos mecanismos financieros, administradores y acomodaticios receptores de recursos externos, por medio de giros en todo sentido pasivos efímeros, elitistas y restringidos que 
no requerían de niveles elevados de participación popular, sino más que todo de capacidad contable, administrativa y legal de las directivas comunales, para el manejo de fondos y obras de infraestructura a cambio de lo cual las comunidades se mantenían reguladas en sus posiciones tradicionales por los poderes financieros, administrativos y políticos de los mandos centrales de la tecnoburocracia pública". (Vega 1982)

En este sentido, cabe plantearse nuevamente la conclusión que nos parece más certera para este periodo del en el desarrollo comunal institucionalizado en Costa Rica:

Una función principal del desarrollo comunal institucionalizado fue acompañar con relativo éxito los intensos procesos de expansión agropecuaria e industrial que experimentó el país en la década de los setentas.

Y ello, a pesar de que:

Las Asociaciones tendieron a politizarse y ser parte del aparato hegemónico del Estado costarricense, particularmente durante la administración Oduber Quirós quien allá, en San Isidro del General, en 1974, decía que él quería gobernar el país con el concurso de mil Asociaciones de Desarrollo (Pacheco 1966)

Las Asociaciones tampoco pudieron organizarse. No fue posible una estructuración eficiente de las Asociaciones en organizaciones de segundo y tercer grado, y en esa misma medida, capaces de sentarse con los representantes del Estado y plantearles lo que ellas pudieran considerar grandes temas del desarrollo nacional.

Hubo ausencia de un "movimiento de desarrollo comunal" estructurado en organizaciones de segundo y tercer grado. Consecuentemente, la imposibilidad de montar o participar en la agenda política cantonal, regional y nacional. Un corolario importante fue que dirigentes comunales con gran liderazgo fueron captados por sus partidos políticos para ser electos munícipes, una tendencia, que como vimos, fue notoria durante la década que abarcó la investigación.

Y no pudieron, durante todo el decenio estudiado, lograr niveles de participación de los vecinos, sobre todo, los más pobres.

\section{REFERENCIAS}

Aguilar H. 1983. Origen y desarrollo del movimiento social urbano en la ciudadela 15 de setiembre de Hatillo. Trabajo de Tesis. Facultad de Ciencias Sociales. Escuela de Antropología y Sociología. Universidad de Costa Rica. San José, Costa Rica.
Alvarenga, P. 2005. De vecinos a ciudadanos. Universidad Nacional, San José, Costa Rica.

BID. 1979. Progreso económico y social en América Latina. Banco Interamericano de Desarrollo. Washington, D.C., EEUU.

Camacho, D. 1979. Debates sobre la teoría de la dependencia y la sociología latinoamericana. Universitaria Centroamericana. San José, Costa Rica.

Campanario, P. \& E. Ritcher. 1974. Superpoblación capitalista en América Latina. Un intento de marginalización del concepto de marginalidad. Revista de Estudios Sociales Centroamericanos 9:37-71.

CEPAL, FAO, OIT, SIDECA, Misión Conjunta de Programación para Centroamérica. 1973. Tenencia de la tierra y desarrollo rural en Centroamérica. EDUCA, San José, Costa Rica.

DINADECO. 1974. Diagnóstico de Asociaciones Seleccionadas. Informe preliminar. Departamento de Programacion y Evaluacion. San José, Costa Rica Documento mimeografiado.

DESAL. 1969. Marginalidad en América Latina. Un ensayo de diagnostico. Herder, Barcelona, España.

González, J. 1977. Desarrollo comunal: acción "cosmética”, concientizadora, desarrollista? , p. 23-26, En: DINADECO: "Reflexiones sobre el desarrollo comunal", Ministerio de Gobernación, DINADECO. Imprenta Nacional, San José Costa Rica.

Gónzalez, J. 1977. Una asistencia técnica en desarrollo comunal. Imprenta Nacional, San José, Costa Rica.

Herrero, F. \& L. Garnier. 1982. El desarrollo de la industria en Costa Rica. EUNA, Heredia, Costa Rica.

Lechner, N. 1977. La cuestión del Estado en el capitalismo dependiente. Revista de Estudios Sociales Centroamericanos 16: 129-164.

Martínez, J. 2000. Luces y sombras: formación y transformación de las políticas sociales en América Latina. Cuadernos de Ciencias Sociales 117.

Oficina de Planificación Nacional y Política Económica. Evolución socioeconómica de Costa Rica: 1950-1980. 1982. EUNED, San José, Costa Rica.

Pacheco, R. 1966. Volver a Liberación. El pensamiento de Daniel Oduber sobre organizaciones comunales y DINADECO. Junio de 1966. Folleto Mimeografiado.

Quesada, W. 1977. Los políticos boicotearon desarrollo comunal en Suplemento "Gentes y Paisajes", Periódico la Nación., sin fecha de publicación, página 10.

Reuben, S. 1982. Capitalismo y crisis económica. Treinta años de desarrollo. Porvenir, San José, Costa Rica.

Rivera, R. 1995. Descentralización y la metáfora de la reforma del Estado. FLACSO, San José, Costa Rica.

Sojo, C. 2000. La relación Estado-sociedad civil en el marco de la globalización. Cuadernos de Ciencias Sociales 114. 
Solano, J. 2001. Educación y desarrollo en América Latina: un análisis histórico-conceptual. EUNA, Heredia, Costa Rica.

Tercero, J. \& H. SALAS. 1986. El desarrollo comunal en Costa Rica. DINADECO, Asociaciones de Desarrollo Comunal. Alternativas de participación popular. Escuela de Historia. Tesis de Grado. Universidad de Costa Rica. San José, Costa Rica.
UNESCO. 1962. Aspectos sociales del desarrollo económico en América Latina. UNESCO, Bruselas, Bélgica.

Vega, J. 1982. Hacia una interpretación del desarrollo costarricense: ensayo sociológico. Porvenir, San José, Costa Rica.

Weber, M. 1944. Economía y sociedad. Esbozo de la sociología comprensiva. Fondo de Cultura Económica, México. 
